\title{
Hypohydrotic Ectodermal Dysplasia in an Indian Family
}

\author{
Anish A. Gupta1', Swati S Gotmare2, Megha Jain1, Treville Pereira² and Pooja Khare
}

\begin{abstract}
Hereditary ectodermal dysplasia (HED) is a rare genetic disorder chiefly affecting ectodermally derived structures including hair, nails, sweat glands etc. with pathognomic manifestations such as hypotrichosis, hypohidrosis, and hypodontia. Hypohidrotic ectodermal dysplaisa, being the most frequently encountered subtype and HED, being the rare subtype. HED is primarily transmitted through X-linked recessive trait in which the gene is carried by the female and manifested in male. Although rare, this disorder may be seen affecting lot of members of the same family. We hereby report a series of four cases with common classical manifestations accompanied with spoon shaped nails, hyperpigmentation, oligodontia and hypotrichosis. The patients were treated for prosthetic rehabilitation and were asked to wear cool clothing.
\end{abstract}

Key Words: Anodontia, Hypotrichosis, Hypohidrosis, Mutation, Sweat glands.

\section{INTRODUCTION}

Ectodermal derivatives include sweat glands, hair, nails, nerves, and detal enamel. Disturbance in any of these ectodermally derived tissues leads to the disorder known as hereditary ectodermal dysplasia (HED). Hypohidrotic ectodermal dysplaisa (HHED) being the most frequent subtype and the other less common is hydrotic ectodermal dysplasia (HyED). ${ }^{1}$ Danz (1792) first encountered a case of HED. Freire-Maia and Pinheiro are credited with the first systematic classification of HED. ${ }^{2}$ Mutations in ectodysplasin A and ectodysplasin A receptor are responsible for HED.

Here, we report four cases of HHED from the same family of Indian descent.

\section{CASE REPORT}

Cases I and II: Two male siblings aged 18 and 16 years respectively reported to our clinic with the chief complaint of spacing between the teeth present in both upper and lower jaws.

Common findings in Cases I and II: On clinical examination, they revealed missing teeth in all quadrants. Their parents were apparently normal, although the mother reported to have missing teeth in early life. According to parents, the siblings had sparse hair in their early

1 Department of Oral Pathology and Microbiology, Peoples Dental Academy, Bhopal, India

2 Department of Oral Pathology and Microbiology, D Y Patil University School of Dentistry, Navi Mumbai, India

3 Department of Oral Medicine and Radiology, Peoples Dental Academy, Bhopal, India

Correspondence: Dr. Anish A Gupta, Department of Oral Pathology and Microbiology, Peoples Dental Academy, Bhopal, India

E-mail:anishpooja687@yahoo.co.in

Received: December 23, 2017; Accepted: October 10, 2018 childhood which increased over time. The eyebrows and eyelashes were scanty. The skin appeared glossy, smooth, thin, and hyper pigmented. The ear lobes were small and attached to the skin of the cheek. They also complained of inability to sweat.

Case I had a hoarse voice which may be due to disturbance in pharyngeal and laryngeal developments. The nails were ill formed with some of them showing free lateral margins flushing with the skin and some of them were spoon shaped (Figure 1).

Case II, in addition to the above mentioned common features, had flat nipples. This patient too showed spoon shaped nails. He also complained of recurrent ulcerations causing severe pain and discomfort.

Case III: One of their 12-year female maternal cousins also had similar features of oligodontia in addition to hypotrichosis involving facial hair, eyebrows and eyelashes. Her first tooth had erupted at the age of 20 months. Radiograph showed many missing permanent tooth germs and also deciduous teeth that had not shed till this age. Anterior teeth were conical shaped (Figure 2). The parents also reported that their first male child had scanty hair in his childhood, but died after 2 years. The reason of his death was uncertain.

Case IV: A 3-year female sibling also had missing teeth and sharp conical teeth. Examination revealed scanty and fine hair on the scalp. The bridge of the nose was depressed. The supraorbital ridges were pronounced with frontal bossing and protuberant lips. Intraoral examination revealed partial absence of teeth and high arched palate (Figure 3). This description was fitting the diagnosis of HHED.

The patients were referred to dermatologist to confirm the findings and the diagnosis of HHED. The patients were treated for prosthetic rehabilitation and were asked to wear cool clothing. 


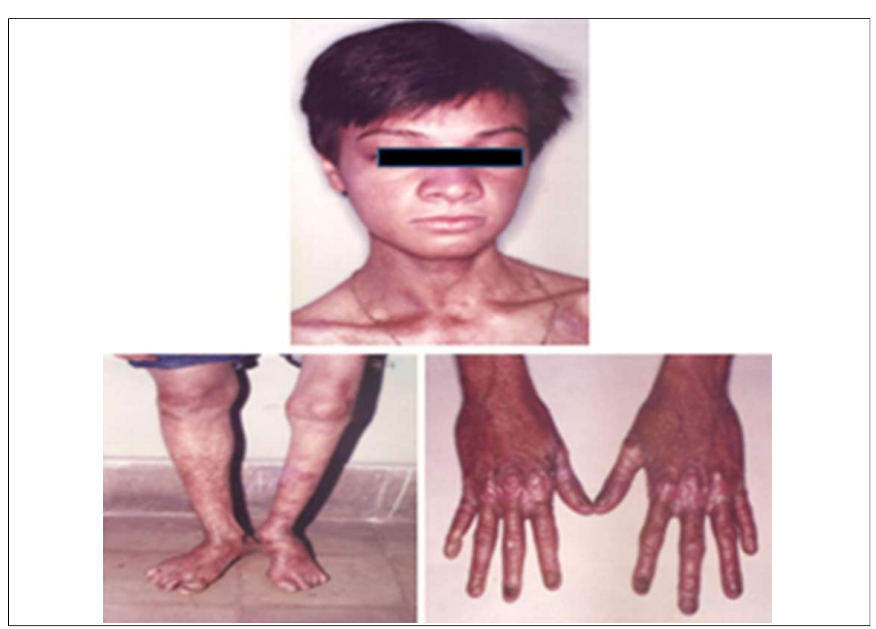

Figure 1: Case I. 18-year boy showing glossy, smooth, shiny, hyperpigmented skin and spoon shaped nails.

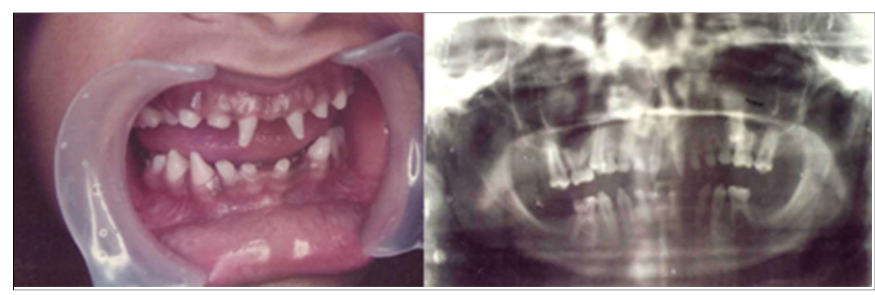

Figure 2: Case III. Intra-oral and radiographic presentation showing multiple missing teeth. Anterior teeth were conical in shape

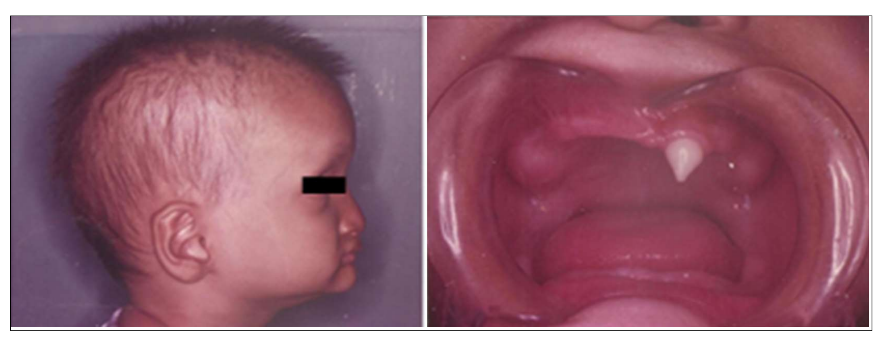

Figure 3: Case IV. Prominent supraorbital ridge, frontal bossing, and Intraoral examination revealed multiple missing teeth.

Written consents were taken from all the patients after informing them about the publication.

\section{DISCUSSION}

HED is a syndrome affecting epidermis and its appendages. Its earliest description dates back to 1848 by John Thurman. Wedderburn reported similar cases in a Hindu family from India in the year 1875. This was the first report which confirmed 10 cases in a span of four generations. ${ }^{3}$ Pinheiro and Freire-Maia uniquely classified ED by giving a specific number to the ectodermal structures involved. Accordingly, numbers given were as follows: 1 for hair, 2 for teeth, 3 for nails, and 4 for sweat glands. Combinations of these formed 10 subgroups. The studies on EDs show that there is a molecular-genetic basis which results in mutations of certain genes resulting in varied clinical findings. Priolo and Laganà proposed a new classification which identified two groups, each consisting of defects in developmental regulation/epithelial mesenchymal interaction and defects in cytoskeleton maintenance and cell stability, respectively. ${ }^{4}$ According to Bani et al., there are two major types of EDs, these include anhidrotic or hypohidrotic (Christ-Siemens-Touraine syndrome) - in which the sweat glands are either completely or partially absent and hydrotic (Clouston's syndrome) - in which sweat glands are normal. 5

EDA is a signaling molecule belonging to tumor necrosis factor family. It has been shown to play a vital role in the development of ectodermally derived tissues. Any mutation in this molecule results in clinical manifestations of ectodermal dysplasia. ${ }^{6}$ Till date, 200 point mutations of EDA and around 40 mutations in EDAR have been reported. ${ }^{7}$

The most dramatic features of this disease is the reduced number of facial hair, due to which there is extreme fragility of the shafts which lack rigidity and reduced number of teeth causing esthetic and functional problems. Depending on the HHED or HyED, there is reduced number of sweat glands and normally functioning sweat glands, respectively. Other features include defective alveolar ridge formation, altered nasal and sebaceous secretions, dry eyes, skin fragility, lack of dermal ridges, hyper pigmentation, rough and scratchy voice, retruded appearance of midface. ${ }^{8}$ The present cases were aged between 3 years to 18 years, belonging to a single family. Although most studies indicate consanguity, ${ }^{9}$ these cases did not show this pattern and were in accordance with. 10 All these cases were diagnosed on the basis of clinical manifestations such as sparse hair, anodontia, reduced sweating; and are seen in almost all the cases in the literature. One out of four patients had raspy voice and spoon shaped nails. These findings, though common, are not a constant feature, suggesting that there may be different levels of inactivation of $\mathrm{X}$-chromosome in cases of heterozygous females. Moreover, as stated by Trzeciak, there could be mutation in TNF receptor associated factor 6 (TRAF 6) leading to mild manifestations in females. ${ }^{7}$ The most constant problem faced by all these patients was multiple missing teeth, posing a social and functional discomfort. These were in accordance to studies conducted by More CB and Puttaraju GH.9,10 These patients showed hyper pigmentation, dental arch abnormalities, and retruded midface. The first three patients were treated orthodontically and given restorations to fulfill the aesthetic and functional demands. So, in those cases, all the typical features of HHED were found and the clinical manifestations confirmed the diagnosis.

\section{REFERENCES}

1. Huang SX, Liang JL, Sui WG, Lin H, Xue W, Chen JJ, et al. EDA mutation as a cause of hypohidrotic ectodermal dysplasia: A case report and review of the literature. Genet Mol Res 2015; 14:10344-51. 
2. Vaidya S, Risbud M, Kshar A, Ramdurg P. Hereditary ectodermal dysplasia: Report of 11 patients from a family. Indian $\mathrm{J}$ Dent Res 2013; 24:502-6.

3. Singh A, Jolly SS, Kaur S. Hereditary ectodermal dysplasia. Br J Dermatol 1962; 74:34-7.

4. Priolo $M$, Laganà $C$. Ectodermal dysplasias: $A$ new clinicalgenetic classification. J Med Genet 2001; 38:579-85.

5. Bani M, Tezkirecioglu AM, Akal N, Tuzuner T. Ectodermal dysplasia with anodontia: A report of two cases. Eur J Dent 2010; 4:215-22.

6. Mikkola ML, Thesleff I. Ectodysplasin signaling in development. Cytokine Growth Factor Rev 2003; 14:211-24.
7. Trzeciak WH, Koczorowski R. Molecular basis of hypohidrotic ectodermal dysplasia: An update. J Appl Genet 2016; 57: 51-61.

8. Wright JT, Grange DK, Richter MK. Hypohidrotic ectodermal Dysplasia. 2003. Available from http://www.ncbi.nlm.nih.gov/ books/NBK1112/. Accessed on 25 April 2017.

9. More CB, Bhavsar K, Joshi J, Varma SN, Tailor M. Hereditary ectodermal dysplasia: A retrospective study. J Nat Sci Biol Med 2013; 4:445-50.

10. Ogunrinde GO, Zubair RO, Ajike SO, Ige SO. Hypohidrotic (anhidrotic) ectodermal dysplasia in female twins. Niger $\mathrm{J}$ Clin Pract 2012; 15:98-100.

.......... 